\title{
Scrutinizing DEEC, TDEEC, BEENISH and IBEENISH Protocol in Heterogeneous Wireless Sensor Network's (WSN's)
}

\author{
V. Baby Shalini
}

\begin{abstract}
WSN's involve abundant sensor's with inadequate power, which will dispatch the monitored information to the Base Station (BS) which needs extra energy. Clustering is a key strategy for enhancing the sensor network lifespan by diminishing the energy consumption. Energy competent clustering practice be supposed to be delineated for heterogeneous WSN. Effectiveness of heterogeneous protocols deteriorates while altering the heterogeneity. In this paper work, Distributed Energy Efficient Clustering (DEEC) was investigated first, at that point Threshold DEEC (TDEEC), Balanced Energy Efficient Network Integrated Super Heterogeneous (BEENISH) and Improved BEENISH (IBEENISH) under more than a few scenarios were examined and then outcomes were compared. In all four cases, Cluster Heads (CH's) were preferred with respect to the remnant energy of sensor's. Observations made thoroughly concerning the performance on the bases of network lifespan and packets received by the BS. IBEENISH performs superior than other existing protocols like DEEC, TDEEC and BEENISH.
\end{abstract}

Keywords: Clustering, heterogeneous, residual energy, cluster head.

\section{INTRODUCTION}

$\mathrm{W}_{\mathrm{SN}}$ [1] involve immense amount of sensor nodes deployed indiscriminately in real world milieu for perceiving the diverse ecological effects like sound, temperature, pressure and so forth. Here, sensor's offer a potent amalgamation of distributed perceiving, computing and communication. The consistently expanding capacities of sensor's facilitate a broad range of applications, for example, military, survival monitoring, battlefield reconnaissance, health care and object tracking etc and in the meantime, abundant challenges like stringent energy constraints, diminutive computational capability are also there. Every sensor node encompasses of four components, a processing unit, a transceiver unit, a power unit and a sensing unit. The architecture of sensor node is depicted in Fig. 1.

Each sensor's in sensing unit is liable for accumulating the information and generate analog signal as output. This signal is subsequently translated in to digital signals by Analog to Digital Converter (ADC) and then feed into processing unit. The microcontroller unit in processing unit is in-charge of

Revised Manuscript Received on December 05, 2019

* Correspondence Author

Baby Shalini*, Information Technology, Kalasalingam Academy of Research and Education, Krishnankoil, India. Email: v.babyshalini@klu.ac.in. administering the other components in the node and it may be of on-board memory or united with a petite storage unit incorporated into the embedded board. The memory unit is mainly for temporal storage of perceived data and it can be Random Access Memory (RAM), Read Only Memory (ROM).

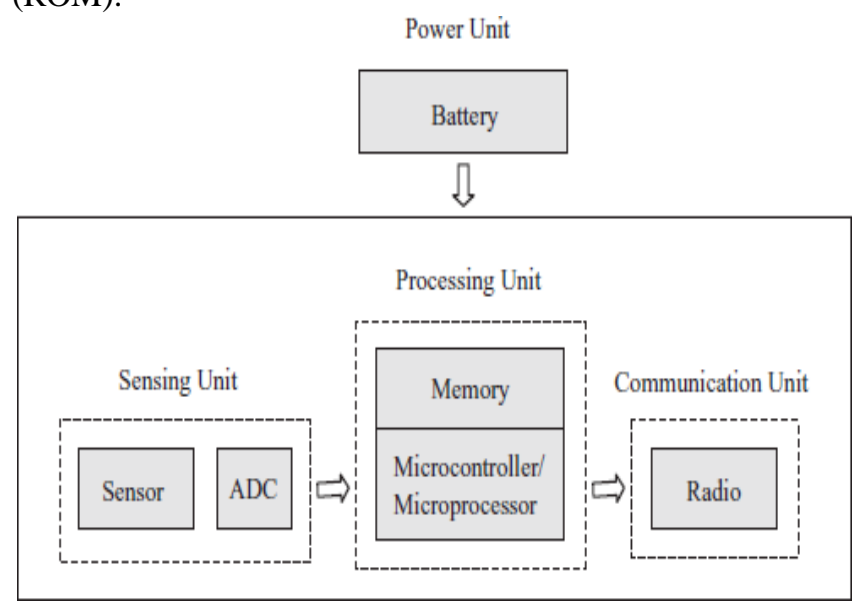

Fig. 1. Structure of Sensor Node

The power unit, which is one of the critical element provides energy to the node. Generally, sensor's are power constrained because of limited battery, in addition to that sensor's are dispersed in hostile situation where it is not viable either to revive or replace their battery. As a result, battery plays the foremost task in WSN. Transceiver unit carry out both transmission as well as reception of observed data with other nodes either in single hop or multi-hop manner. In this process, most of the energy is devoured for transmitting the data from sensor's to the BS. To overcome this crisis, cluster based protocols $[7,11]$ have be a focus for a group of researchers. Clustering [2,3] consist of dual phases: setup phase followed by steady state phase. In the former phase, whole network is split in to clusters (collection of nodes). In each one of the cluster, there is a node that will function as a $\mathrm{CH}$ and leftover nodes will behave as a cluster members (CM). In latter phase, members in the cluster will observe the milieu and pass on the data to $\mathrm{CH}$ systematically. The key benefit of clustering is to diminish the transmission distance by conveying the data with $\mathrm{CHs}$ [6] over relatively petite distances.

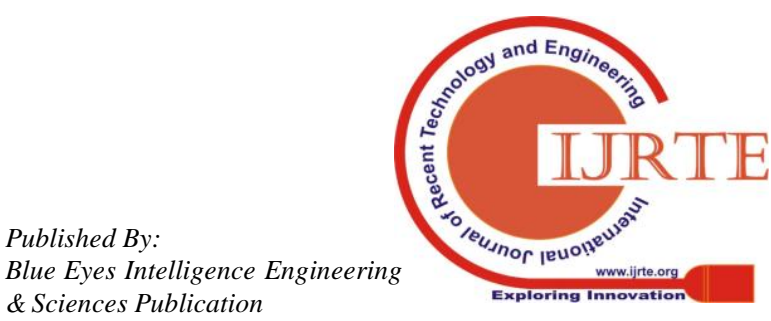


There are two varieties of networks somewhere clustering strategy can be practiced. Nodes with identical energy is known as homogenous WSN and nodes with dissimilar energy is known as heterogeneous WSN [9]. Although the majority clustering $[4,5]$ algorithm mostly considers the residual energy of node while selecting $\mathrm{CH}$. To further perpetuate the existence of the network, WSNs with heterogeneity [8,10] was developed. DEEC [12-14], TDEEC [15], BEENISH [16] and IBEENISH [17] devised for heterogeneous sensor network. DEEC is a bi level heterogeneous WSN consist of normal nodes along with advanced nodes with superfluous energy. TDEEC is a three level heterogeneous network have normal, advanced as well as super nodes wherever super nodes include utmost energy in contrast to rest of the sensors. BEENISH is a four level heterogeneous network which consist of normal, advanced, super and ultrasuper sensor's whereas ultrasuper sensor's have surplus energy comparing to remaining modes. IBEENISH is a five level heterogeneous network include normal, intermediate, advanced, super and ultrasuper nodes whereas ultrasuper nodes have utmost energy in comparison to rest of the nodes. It is found that diverse protocols have dissimilar efficiency in terms of stability period, network lifespan as well as packets received by the BS.

Paper Organization: The rest of the paper is structured as follows. In Section II, various earlier works related to heterogeneous protocol was discussed. Section III illustrates the model of heterogeneous WSN. Section IV presents the outline of Heterogeneous WSN Protocols. Section V compares the results of variety of heterogeneous protocol by simulation. Finally, Section VI elaborates the conclusion.

\section{RELATED WORKS}

Qing et al. [14] described a two level heterogeneous Distributed Energy Efficient Clustering (DEEC) algorithm with which CH's are preferred on the bases of proportion of left over energy of every node to average energy of the sensor network. Here, $\mathrm{CH}$ was revolved among all sensor's to balance the energy consistency. The major shortcoming is advanced nodes will be penalized repeatedly in comparison to normal nodes.

Parul Saini et al. [15] put forward a three level heterogeneous Threshold DEEC (TDEEC) protocol in which the value of threshold is adjusted in accordance with which a sensor will make a decision to turn into as a $\mathrm{CH}$ or not, based on the relative amount of remaining energy and average network energy of that particular round in respect to the optimum quantity of CH's.

Qureshi et al. [16] demonstrated a four level BEENISH whereupon CH's are decided on the bases of left over energy level of sensors.

Harwinderpal Singh et al. [17] elaborated a five level heterogeneous Improved BEENISH (IBEENISH) protocol where in CH's have chosen based on residuary energy of the sensor's.

\section{MODEL OF HETEROGENEOUS WSN}

\section{A. Radio Model}

Network Existence is very much get affected by the energy model utilized for simulation. The first order radio energy model is delineated in Fig. 2. Here K-bit packet is conveyed up to a distance d.

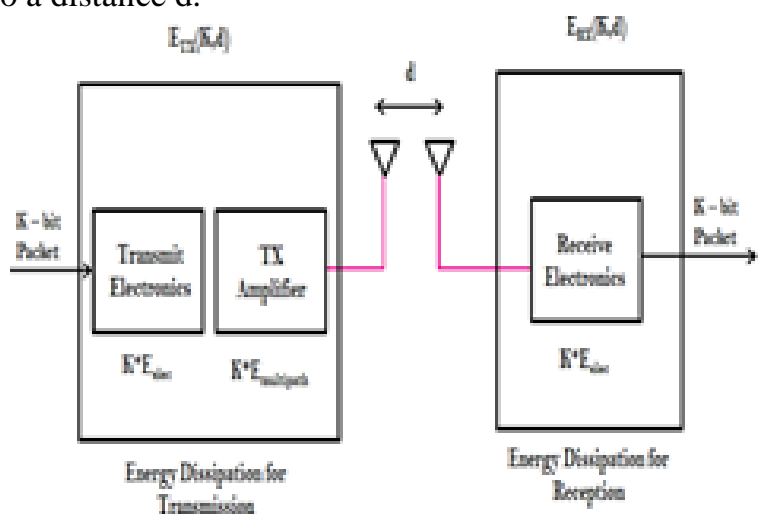

Fig. 2. Radio Model

Transmission energy $E_{T X}$ signify the transmitter of a sensor node which disburse energy for delivery of K-bit packet upon a distance of $d$ meters not as much as and greater than threshold distance $t d_{0}$ is given as

$$
\begin{aligned}
& E_{T X}(K, d)=K *\left(E_{\text {elec }}+d^{2} * E_{f s}\right) \\
& E_{T X}(K, d)=K *\left(E_{\text {elec }}+d^{4} * E_{m p}\right)
\end{aligned}
$$

Here, $E_{\text {elec }}$ is the energy dissipation of radio for functioning the transceiver circuit, $E_{f s}$ and $E_{m p}$ is the energy expended in free space and multipath model respectively. In reception of $\mathrm{K}$ message, the energy that a node dissipates at a distance of d meters is specified as

$E_{R X}(K, d)=K * E_{\text {elec }}$

Threshold distance $t d_{0}$ is reckoned with the help of the formula

$$
t d_{0}=\frac{E_{f s}}{E_{m p}}
$$

Energy expended by the network for every round $\mathrm{r}$ is

$$
E_{\text {Expended }}=K\left(\begin{array}{l}
2 N E_{\text {elec }}+N E_{D A} \\
+\varepsilon E_{m p} d_{C H t o B S}^{4}+N \varepsilon E_{f s} d_{C M t o C H}^{2}
\end{array}\right)
$$

Where, $\varepsilon$ is the total quantity of $\mathrm{CH}$ be active in the network for a particular round, $d_{C H t o B S}$ as well $d_{C H t o C H}$ is the transmittal distance from $\mathrm{CH}$ to $\mathrm{BS}$ and $\mathrm{CM}$ to $\mathrm{CH}$ respectively and can be evaluated using the following equations

$$
\begin{aligned}
& \varepsilon=\sqrt{\frac{N}{2 \Pi} * t d_{0}} * \frac{M}{d_{C M t o C H}} \\
& d_{C H t o B S}=0.765 * \frac{M}{2} \\
& d_{C M t o C H}=\frac{M}{\sqrt{2 * \Pi * \varepsilon}}
\end{aligned}
$$




\section{B. Heterogeneous WSN Model}

Here, $N$ sensor's are dispersed haphazardly in a square region with dimension $M \times M$ and BS is situated at the midpoint of the sensing region is revealed in Fig. 3.

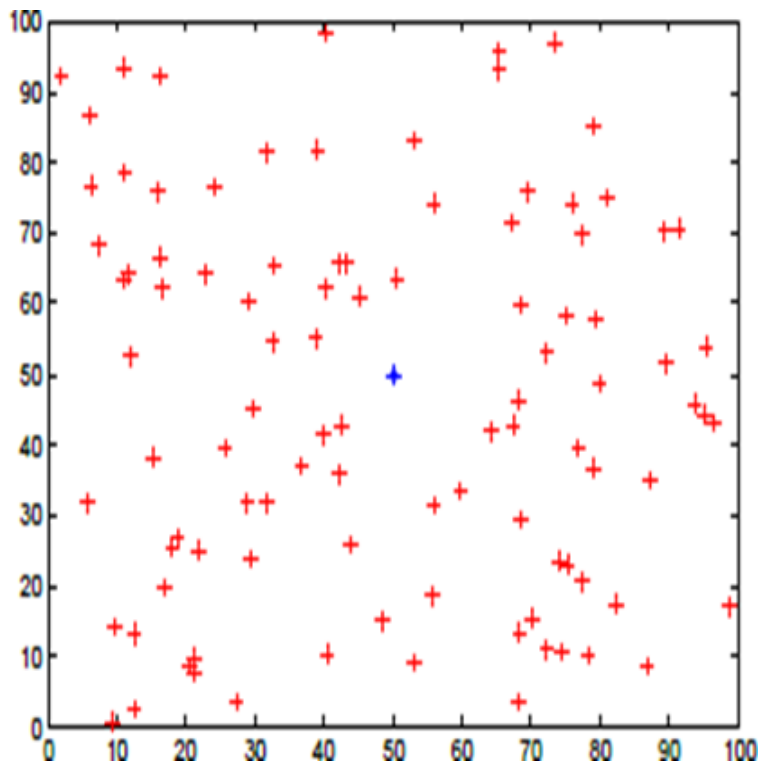

Fig. 3. Random Wireless Sensor Model

Sensor nodes with heterogeneity (i.e diverse energy level) is said to be Heterogeneous WSNs.

Two Level Heterogeneous WSN Model

In this model, $E_{0}(1+a 1)$ energy is incorporated in $m N$ advanced nodes (i.e these sensor's get hold of $a 1$ times superfluous energy in comparison to normal nodes) and $(1-m) N$ normal nodes equipped with $E_{0}$.

The entirety primary energy for two level networks is given by

$$
E_{\text {Total }}=N E_{0}(1+a 1 m)
$$

Three Level Heterogeneous WSN Model

Here, $\mathrm{Nmm}_{0}$ is total amount of super nodes comprise energy of $E_{0}(1+b 1)$ which have $b 1$ times superfluous energy as well as $N m\left(1-m_{0}\right)$ is entire quantity of advanced sensor's with energy $E_{0}(1+a 1)$ which possess a1 times additional energy when comparing to normal sensor's and $(1-m) N$ is the whole amount of normal nodes with $E_{0}$.

The whole amount of initiatory energy for three level network is specified by

$$
E_{\text {Total }}=N E_{0}\left(1+a 1 m\left(1-m_{1}\right)+m m_{1} b 1\right)
$$

Here, $N m m_{0} m_{1}$ is total quantity of ultrasuper sensor's with energy $E_{0}(1+c 1)$ which have possession of $c 1$ times additional energy, $\operatorname{Nmm}_{0}\left(1-m_{1}\right)$ is entirety quantity of super nodes with energy $E_{0}(1+b 1)$ which own $b 1$ times extra energy, $N m\left(1-m_{0}\right)$ is the total number of advanced nodes with energy $E_{0}(1+a 1)$ which own $a 1$ times more energy than normal nodes and $N(1-m)$ is the total number of normal nodes with energy $E_{0}$.

The total initial energy of the four level network is given by, $E_{\text {Total }}=N E_{0}\left(\begin{array}{l}1+m a 1\left(1-m_{0}\right) \\ +m m_{0} b 1\left(1-m_{1}\right)+m m_{0} m_{1} c_{1}\end{array}\right)$

Five Level Heterogeneous WSN Model

Here, $N m m_{0} m_{1} m_{2}$ is total amount of ultrasuper nodes hold energy of $E_{0}(1+d 1)$ i.e nodes have d1 times superfluous energy, $N m m_{0} m_{1}\left(1-m_{2}\right)$ is entire quantity of super nodes with energy $E_{0}(1+c 1)$ which be in possession of $c l$ times additional energy, $\mathrm{Nmm}_{0}\left(1-m_{1}\right)$ is the total amount of advanced nodes with energy $E_{0}(1+b 1)$ which own $b 1$ times extra energy, $N m\left(1-m_{0}\right)$ is the total amount of intermediate nodes include energy of $E_{0}(1+a 1)$ which own $a 1$ times additional energy as compared to normal nodes as well as $N(1-m)$ is the entire amount of normal sensor's hold $E_{0}$.

The initiatory energy of the five level network is given by,

$$
E_{\text {Total }}=N E_{0}\left(\begin{array}{l}
1+m a 1\left(1-m_{0}\right)+m m_{0} b 1\left(1-m_{1}\right) \\
+m m_{0} m_{1} c_{1}\left(1-m_{2}\right)+m m_{0} m_{1} m_{2} d 1
\end{array}\right)
$$

\section{OUTLINE OF HETEROGENEOUS WSN PROTOCOLS}

In all protocol's, CH's are chosen on the bases of proportion of residuary energy of a sensor $\left(E_{\text {res }}\right)$ and network average energy $\left(E_{a}\right)$ for each round $\mathrm{r}$.

$E_{a}$ is found using the formula

$$
E_{a}=\frac{1}{N} * \text { TotalEnergy } *\left(1-\frac{r}{N E}\right)
$$

Where $N E$ is the network existence and is calculated as

$$
N E=\frac{\text { TotalEnergy }}{E_{\text {Expended }}}
$$


Scrutinizing DEEC, TDEEC, BEENISH and IBEENISH Protocol in Heterogeneous Wireless Sensor Network's (WSN's)

\section{A. DEEC}

The probabilities of normal as well as advanced nodes are as follows

$$
p(i)=\left\{\begin{array}{c}
\frac{p_{i} E_{\text {res }}}{(1+a 1 m) E_{a}} \forall \text { normal_nodes } \\
\frac{p_{i}(1+a 1) E_{\text {res }}}{(1+a 1 m) E_{a}} \forall \text { advanced_nodes }
\end{array}\right.
$$

\section{B. TDEEC}

The probabilities of normal, advanced and super nodes are as follows

$$
p(i)=\left\{\begin{array}{l}
\frac{p_{i} E_{\text {res }}}{\left(\begin{array}{l}
1+a 1 m\left(1-m_{1}\right) \\
+m_{1} b 1
\end{array}\right) E_{a}} \\
\frac{p_{i}(1+a 1) E_{\text {res }}}{\left(\begin{array}{l}
1+a 1 m\left(1-m_{1}\right) \\
+m m_{1} b 1
\end{array}\right) E_{a}} \forall \text { advanced_nodes } \\
\frac{p_{i}(1+b 1) E_{\text {res }}}{\left(\begin{array}{l}
1+a 1 m\left(1-m_{1}\right) \\
+m m_{1} b 1
\end{array}\right) E_{a}} \forall \text { super_nodes }
\end{array}\right.
$$

\section{BEENISH}

The probabilities of normal, advanced, super as well as ultrasuper nodes are as follows

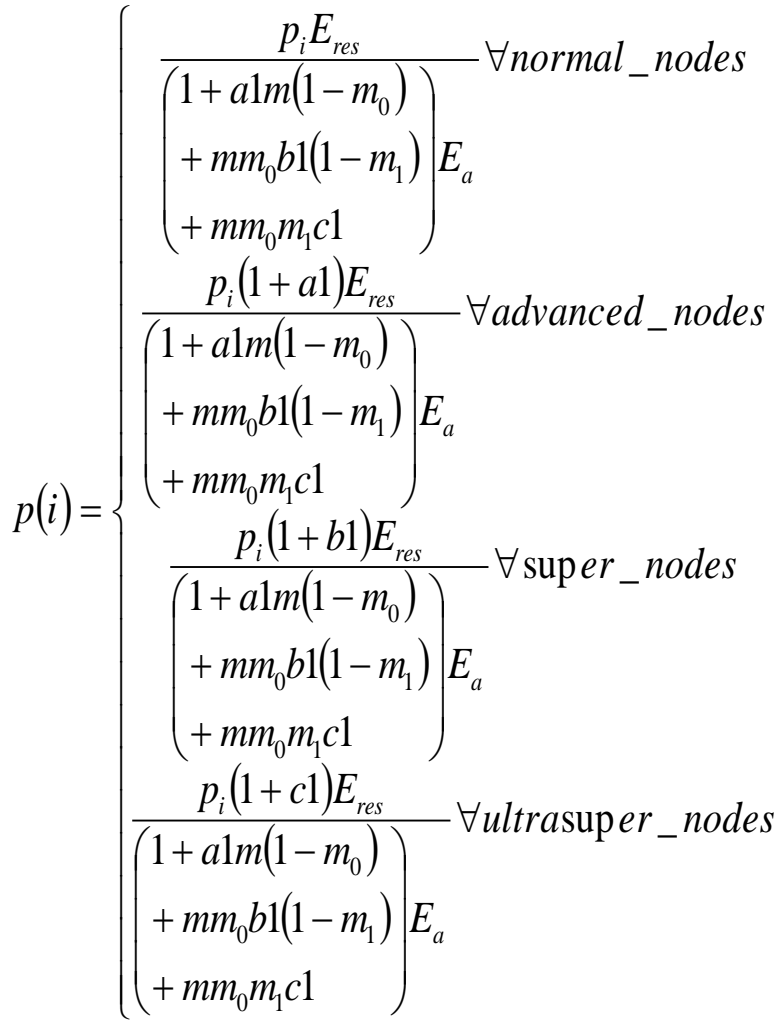

\section{IBEENISH}

The probabilities of normal, intermediate, advanced, super and ultrasuper nodes are as follows

$$
\begin{aligned}
& \int \frac{p_{i} E_{\text {res }}}{\left(\begin{array}{l}
1+a 1 m\left(1-m_{0}\right)+m m_{0} b 1\left(1-m_{1}\right) \\
+m m_{0} m_{1} c 1\left(1-m_{2}\right)+m m_{0} m_{1} m_{2} d 1
\end{array}\right) E_{a}} \forall \text { normal_nodes } \\
& \frac{p_{i}(1+a 1) E_{\text {res }}}{\left(\begin{array}{l}
1+a 1 m\left(1-m_{0}\right)+m m_{0} b 1\left(1-m_{1}\right) \\
+m m_{0} m_{1} c 1\left(1-m_{2}\right)+m m_{0} m_{1} m_{2} d 1
\end{array}\right)} \forall \text { int ermediate_nodes }
\end{aligned}
$$

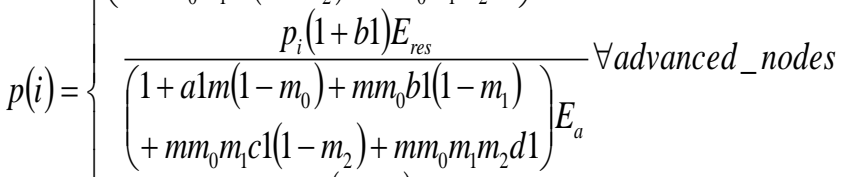

$$
\begin{aligned}
& \frac{p_{i}(1+c 1) E_{\text {res }}}{\left(\begin{array}{l}
1+a 1 m\left(1-m_{0}\right)+m m_{0} b 1\left(1-m_{1}\right) \\
+m m_{0} m_{1} c 1\left(1-m_{2}\right)+m m_{0} m_{1} m_{2} d 1
\end{array}\right) E_{a}} \forall \text { super_nodes } \\
& \frac{p_{i}(1+d 1) E_{\text {res }}}{\left(\begin{array}{l}
1+a 1 m\left(1-m_{0}\right)+m m_{0} b 1\left(1-m_{1}\right) \\
+m m_{0} m_{1} c 1\left(1-m_{2}\right)+m m_{0} m_{1} m_{2} d 1
\end{array}\right) E_{a}} \forall \text { ultrasuper_nodes }
\end{aligned}
$$

In all heterogeneous protocols, threshold is altered on the bases of proportion of $E_{\text {res }}$ and $E_{a}$ in respect to $\varepsilon$. The threshold is as follows

$$
T(s)=\frac{p}{1-p\left(r \bmod \frac{1}{p}\right)} * \frac{E_{r e s} * \varepsilon}{E_{a}}
$$

\section{RESULT AND DISCUSSION}

In this section, the experimental outcomes of DEEC, TDEEC, BEENISH and IBEENISH with MATLAB was assessed. The radio and other parameters utilized for simulation is exhibited in Table I \& II.

Table- I: Radio Parameters

\begin{tabular}{|c|c|}
\hline Parameter & Value \\
\hline $\begin{array}{c}\text { Transmitter and Receiver Electronics }(\mathrm{E} \\
\text { elec })\end{array}$ & $50 \mathrm{nj} / \mathrm{bit}$ \\
\hline $\begin{array}{c}\text { Free Space Model }\left(E_{f s}\right) \\
10 \mathrm{pj} / \mathrm{bit} / \mathrm{m}^{2}\end{array}$ & $0.0013 \mathrm{nj} / \mathrm{bit} / \mathrm{m}$ \\
\hline $\begin{array}{c}\text { Multi-path Model }\left(E_{a m p}\right) \\
\text { Fusion } \\
\left(E_{D A}\right)\end{array}$ & $5 \mathrm{nj} / \mathrm{bit} / \mathrm{message}$ \\
\hline Threshold Distance $\left(\mathrm{td}_{0}\right)$ & 80 meters \\
\hline
\end{tabular}

Table- II: Other Parameters

\begin{tabular}{|c|c|}
\hline Parameter & Value \\
\hline Number of Sensor Nodes & 100 \\
\hline Network Area(MxM) & $100 \times 100$ \\
\hline Probability to become Cluster Head & 0.1 \\
\hline Sensor Node Initiatory Energy E 0 & $0.5 \mathrm{~J}$ \\
\hline Information Size $(\mathrm{K})$ & 4000 bits \\
\hline
\end{tabular}


The parameters exploited to find out the performance of WSN are as follows:

1) Stability Period

Is defined as period from initiation of sensor network operation upto demise of first sensor.

2) Network Existence

Is defined as time bound from the commence of sensor network operation and after the demise of final alive sensor.

3) Packets Received by the BS

Is the enumerate of number of packets (In bits) were collected by end user.

In DEEC, network incorporates 30 normal nodes with primary energy along with 70 advanced nodes with one-time extra energy as compared with normal nodes. In TDEEC, network accommodates 30 normal nodes with original energy, 28 advanced nodes with one-time additional energy as well as 42 super sensor's with 1.5 times surplus energy as compared to normal sensor's. In BEENISH, a network involves 30 normal sensor's with primary energy, 28 advanced sensor's with one time more energy, 21 super nodes with 1.5 times additional energy as well as 21 ultrasuper nodes with 2 times more energy in contrast with normal nodes. In IBEENISH, network includes 30 normal nodes with elementary energy, 28 intermediate nodes with one-time superfluous energy, 21 advanced nodes with 1.5 times more energy, 13 super nodes with 2 times extra energy and 8 ultrasuper nodes with 2.5 times additional energy than normal nodes.

Table- III: Stability Period

\begin{tabular}{|c|c|c|}
\hline $\begin{array}{c}\text { SI. } \\
\text { No }\end{array}$ & Protocol & $\begin{array}{c}\text { Stability Period } \\
\text { (In Rounds) }\end{array}$ \\
\hline 1 & DEEC & 1343 \\
\hline 2 & TDEEC & 1542 \\
\hline 3 & BEENISH & 1784 \\
\hline 4 & IBEENISH & 1961 \\
\hline
\end{tabular}

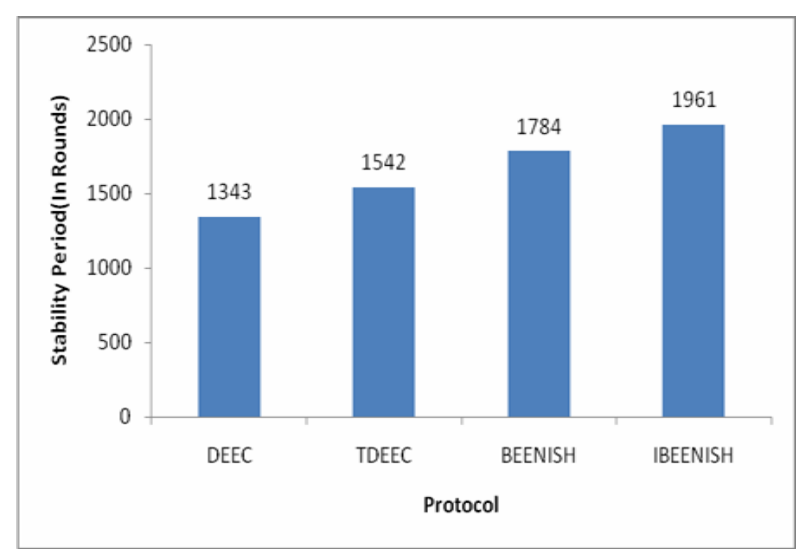

Fig. 4. Stability Period

Fig. 4 depicts the round number at which first node dies. The first sensor dies at 1343, 1542, 1784 and 1961 rounds respectively for algorithms like DEEC, TDEEC, BEENISH and IBEENISH.
Table- IV: Network Lifetime

\begin{tabular}{|c|c|c|}
\hline Sl. No & Protocol & Network Lifetime (In Rounds) \\
\hline 1 & DEEC & 4989 \\
\hline 2 & TDEEC & 6167 \\
\hline 3 & BEENISH & 7091 \\
\hline 4 & IBEENISH & 8134 \\
\hline
\end{tabular}

Fig. 5 depicts the round number at which the entire nodes die. In DEEC, TDEEC, BEENISH and IBEENISH, network can operate up to $4989,6167,7091$ and 8134 rounds respectively.

Table- V: Packets Received by BS

\begin{tabular}{|c|c|c|}
\hline Sl. No & Protocol & $\begin{array}{c}\text { Packets Received by the BS } \\
\text { (In Bits) }\end{array}$ \\
\hline 1 & DEEC & 334967 \\
\hline 2 & TDEEC & 365411 \\
\hline 3 & BEENISH & 374822 \\
\hline 4 & IBEENISH & 444917 \\
\hline
\end{tabular}

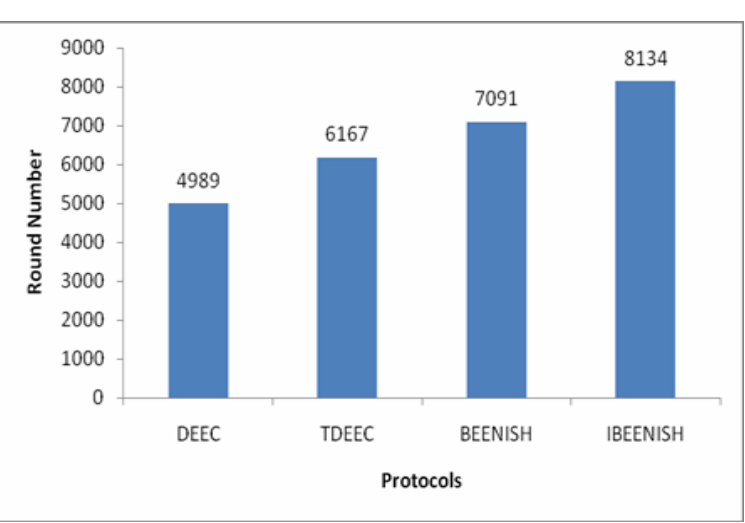

Fig. 5. Network Lifetime

Fig. 6 depicts the total quantity of bits accumulated by the BS.

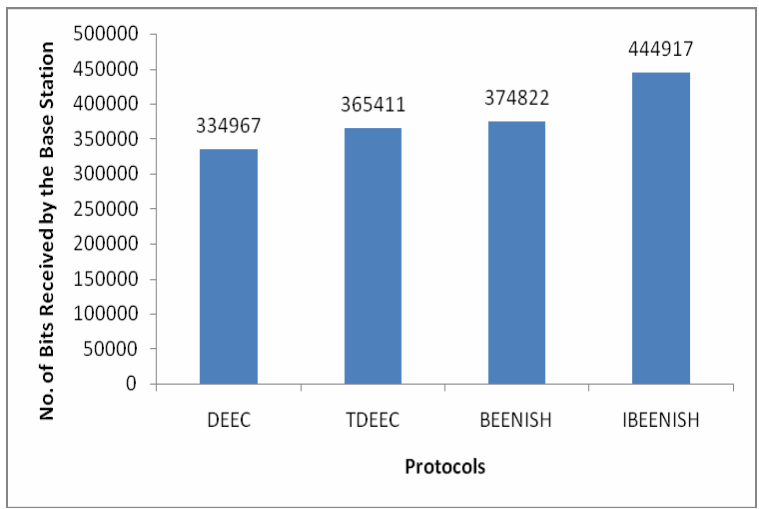

Fig. 6. Numbers of Bits Received by BS

\section{CONCLUSION}

In this paper, DEEC, TDEEC, BEENISH and IBEENISH protocols for heterogeneous WSN was designed and implemented. Here, every sensor's will autonomously choose themselves as a $\mathrm{CH}$ on the bases of its remaining energy. To manage the energy expenditure of sensor's, protocol make use of sensor network average energy as the reference energy. 
Simulation outcomes demonstrate that IBEENISH accomplish better results when compared to DEEC, TDEEC and BEENISH in heterogeneous milieu for WSN.

\section{REFERENCES}

1. I. F. Akyildiz, W. Su, Y. Sankarasubramaniam and E. Cayirci, "Wireless Sensor Networks: A Survey", Computer Networks, 38(4), 2002, 393-422.

2. V. Mhatre and C. Rosenberg, "Design Guidelines for Wireless Sensor Networks: Communication, Clustering and Aggregation", Ad Hoc Network, 2(1), 2004, 45-63.

3. A. A. Abbasi and M. Younis, "A Survey on Clustering Algorithms for Wireless Sensor Networks", Computer Communications, 30 (2007), 2826 -2841 .

4. M. Bsoul, A. A. Khasawneh, A. E. Abdallah, E. E. Abdallah and I. Obeidat, "An Energy-Efficient Threshold-Based Clustering Protocol for Wireless Sensor Networks", Wireless Personal Communication, 70(1), 2013, 99-112.

5. A. U. R. Khan, S. A. Madani, K. Hayat, S. U. Khan, "Clustering Based Power Controlled Routing for Mobile Wireless Sensor Networks", International Journal of Communication Systems, 25(4), 2012, 529-542.

6. A. Wang, D. Yang, D. Sun, "A Clustering Algorithm Based on Energy Information and Cluster Heads Expectation for Wireless Sensor Network", Computer and Electrical Engineering, 38(3), 2012, 662-671.

7. D. Wei, Y. Jin, S. Vural, K. Moessner and R. Tafazolli, "An Energy Efficient Clustering Solution for Wireless Sensor Networks", IEEE Transactions on Wireless Communication, 10(11), 2011, 3973-3983.

8. F. A. Aderohunmu, J. D. Deng and M. K. Purvis, "Enhancing Clustering in Wireless Sensor Networks with Energy Heterogeneity", International Journal of Business Data Communication and Networking, 7(4), 2011, $18-32$.

9. S. Singh, S. Chand and B. Kumar, "Multilevel Heterogeneous Network Model for Wireless Sensor Networks", Telecommunication Systems, 64(2), 2016, 259-277.

10. D. Kumar, T. C. Aseri and R. B. Patel, "EEHC: Energy Efficient Heterogeneous Clustered Scheme for Wireless Sensor Networks", Computer Communications, 32(4), 2009, 662-667.

11. D. Kumar, "Performance Analysis of Energy Efficient Clustering Protocols for Maximizing Lifetime of Wireless Sensor Networks", IET Wireless Sensor Systems, 4(1), 2014, 9-16.

12. S. Singh, A. Malik, R. Kumar, " Energy Efficient Heterogeneous DEEC protocol for Enhancing Lifetime in WSN's", Engineering Science and Technology, an International Journal, 20(1), 2017, 345-353.

13. A. Chamam and S. Pierr, "A Distributed Energy Efficient Clustering Protocol for Wireless Sensor Networks", Journal of Computers and Electrical Engineering, 36(2), 2010, 303-312.

14. L. Qing, Q. Zhu and M. Wang, "Design of a Distributed Energy Efficient Clustering Algorithm for Heterogeneous Wireless Sensor Networks", Computer Communications, 29(12), 2006, 2230-2237.

15. Parul Saini and Ajay K Sharma, "Energy Efficient Scheme for Clustering Protocol Prolonging the Lifetime of Heterogeneous Wireless Sensor Networks", International Journal of Computer Applications, 6(2), 2010,30-36.

16. T. N. Qureshi, N. Javaid, A. H. Khan, A. Iqbal, E. Akhtar and M. Ishfaq, "BEENISH:Balanced Energy Efficient Network Integrated Super Heterogeneous Protocol for Wireless Sensor Networks", Procedia Computer Science, 2013.

17. Harwinderpal Singh and Lokesh Pawar, "IBEENISH: Improved Balanced Energy Efficient Network Integrated Super Heterogeneous Protocol for Wireless Sensor Networks", International Journal of Computer Science and Network, 4(4), 2015, 564-568.

\section{AUTHORS PROFILE}

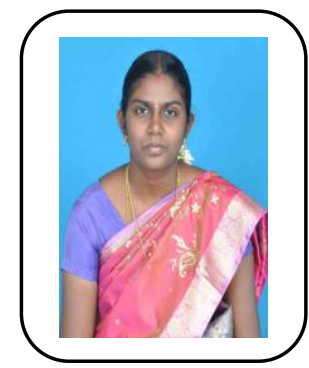

V. Baby Shalini is currently serving as assistant professor in department of Information Technology,Kalasalingam Academy of Research and Education, Krishnankoil..She received her $\mathrm{Ph} . \mathrm{D}$ in 2019. She received her under graduate and post graduate degree in Electronics and Communication Engineering and Communication Systems from Anna University in 2006 and 2008 respectively. She has published some papers in Reputed International Journals and also a Lifetime member of Indian Society of Technical Education(ISTE). 